\title{
Atmospheric close approaches with the Earth considering drag and lift forces
}

\author{
Vivian Martins Gomes ${ }^{1}$ • Jhonathan O. Murcia Piñeros ${ }^{2}$. \\ Antonio Fernando Bertachini de Almeida Prado ${ }^{2}$. Justyna Golebiewska ${ }^{3}$
}

Received: 4 February 2015 / Revised: 5 June 2015 / Accepted: 6 July 2015 / Published online: 24 July 2015 (C) SBMAC - Sociedade Brasileira de Matemática Aplicada e Computacional 2015

\begin{abstract}
A maneuver called "Aero-Gravity Assisted" is known in the literature to increase the energy gains given by a close approach between a spacecraft and a planet using the atmosphere of the planet. In a sequence of studies related to this problem, the present paper studies close approaches between a spacecraft and the Earth, in situations where the passage is close enough to the surface of the Earth such that the spacecraft crosses its atmosphere. The dynamical model considers the atmosphere of the Earth, in terms of drag and lift, the gravitational fields of the Earth and the Sun, assumed to be points of mass, and the spacecraft. The Earth and the Sun are assumed to be in circular coplanar orbits around their common center of mass. The equations of motion are the ones given by the circular planar restricted three-body problem with the addition of the forces generated by the atmospheric drag and lift. The primary objective is to map the variations of energy of the orbits of the spacecraft due to this close approach. The results show how the atmosphere affects the trajectory of the spacecraft, generating situations where the variation of energy changes sign with respect to the gravity part of the maneuver or where they have a zero net result, based in the equilibrium
\end{abstract}

Communicated by Elbert E. N. Macau, Antônio Fernando Bertachini de Almeida Prado and Cristiano Fiorilo de Melo.

\footnotetext{
$\bowtie$ Vivian Martins Gomes

vivianmartinsgomes@gmail.com

Jhonathan O. Murcia Piñeros

jhonathan.pineros@inpe.br

Antonio Fernando Bertachini de Almeida Prado

antonio.prado@inpe.br

Justyna Golebiewska

jg@amu.edu.pl

1 Grupo de Dinâmica Orbital e Planetologia, Universidade Estadual Paulista-FEG/UNESP, Guaratinguetá, Brazil

2 Instituto Nacional de Pesquisas Espaciais-INPE, São José dos Campos, Brazil

3 Faculty of Physics, Astronomical Observatory Institute, Adam Mickiewicz University, Poznan, Poland
} 
between atmospheric and gravity forces. This result opens the possibility of changing only the eccentricity of the orbit, keeping fixed its semi-major axis.

Keywords Astrodynamics · Swing-by - Orbital maneuvers · Aero-gravity maneuver $\cdot$ Close approach

Mathematics Subject Classification 37N05 · 70F07 · 70F15

\section{Introduction}

The close approach maneuver is a well-known technique used in space missions to change the energy of a spacecraft and there are many papers on this topic in the literature. A detailed description of this technique can be found in Broucke (1988), where all the parameters and effects are shown in details.

The most common objective of this type of maneuver is to save fuel in orbital maneuvers, but other situations may occur, like finding a proper timing for a mission. This phenomenon was known long ago by the astronomers that noticed it in the motion of natural celestial bodies, like in problems involving three large bodies (Szebehely 1973; Laskar and Marchal 1984) or the passage of a planet by a rotating star (Ivanov and Papaloizou 2011). In terms of practical applications in Astrodynamics, this phenomenon is also very important. There is a large number of missions that used this technique. A well-known mission was the Voyager spacecraft that traveled to the giant planets of the Solar System using a series of close approaches (Minovich 1961; Flandro 1966) to complete its mission. Later, some other options to visit those same planets and/or their moons were considered, and they can be found in several references (D'Amario et al. 1982; Strange and Longuski 2002; McConaghy et al. 2003; Okutsu et al. 2006; Helton et al. 2002). Similar studies are also available considering close approaches with the interior planets of the Solar System (Hollister and Menning 1970; Titus 1965; Gillespie and Ross 1967), the Moon (Kawaguchi et al. 1995) and Pluto (Longuski and Williams 1991). Some other types of missions using this technique are also available, like making a three-dimensional close approach with the planet Jupiter to make a large modification of the inclination of the orbital plane of the spacecraft (Carvell 1986) or studying sequences of close approaches by the Moon to give more energy to the spacecraft (Dunham and Davis 1985; Marsh and Howell 1988). The literature also shows some other studies related to this problem when considering more complex maneuvers, like combining it with impulsive maneuvers (Prado 1997; Schulz et al. 2002; Silva et al. 2013a, b, 2015) or applying to cloud of particles (Gomes and Prado 2010).

The idea of the present paper was to study the effects of the atmosphere of the Earth in this type of maneuver to consider situations where the approach is close enough to the Earth. This concept is known in the literature as "Aero-Gravity Assisted Maneuver" and there are many studies available on this topic. In particular, the use of "Aero-Gravity Assisted Maneuver" in Mars and/or Venus using higher values for the lift/drag ratio to go to more distant planets, in particular Pluto, or even the Sun, are considered in many publications (McRonald and Randolph 1992; Lewis and McRonald 1992; Bonfiglio et al. 2000; Sims et al. 1995, 2000; Lohar et al. 1997; Randolph and McRonald 1992). The consideration of the shape and/or structure of the spacecraft is also studied (Armellin et al. 2006, 2007). Optimization techniques have also being used in the planar version of the problem (Lohar et al. 1994) or in the three-dimensional case (Lohar 1996). Mission design using this concept and 
multi-objective evolutive optimization can be found in Lavagna et al. (2005). An interesting analytical approach is used in Elices (1995), deriving equations to obtain the maximum effect of this close approach.

Prado and Broucke (1995) and Gomes et al. (2013) study this problem in a similar way, but assuming that the drag is the only force generated by the atmosphere. Considering these information, the contribution of the present paper is a more general study of this problem, to include situations where it is possible to remove energy from the spacecraft, always considering the inclusion of the lift force, which is the force perpendicular to the direction of motion of the spacecraft. It is the same force that makes airplanes to fly. Situations where it is interesting to remove energy may occur when a spacecraft is coming back to the Earth from the exterior Solar System or when the objective of the mission is to send a spacecraft to the interior of the Solar System or to the Sun. In this case, the spacecraft can be launched from the Earth in a interplanetary trajectory that makes a close approach with the Earth after some time, then decreasing its two-body energy with respect to the Sun to go to the direction of the interior of the Solar System. This type of maneuver can also be made with other planets, like Jupiter, to start a trajectory that goes back to the Earth after finishing its mission in Jupiter.

The equations of motion are the ones given by the restricted planar circular three-body problem (Szebehely 1967) with the addition of the atmospheric drag and lift forces. After writing the equations of motion, they are numerically integrated with the initial conditions at the perigee, in the positive and negative senses of time, until a point where the spacecraft reaches a distance that can be considered far enough from the Earth, such that the system Sun-spacecraft can be modeled by the two-body dynamics. At those two points, one for each direction of time, all the information required to obtain the two-body energy Sunspacecraft before and after the close approach is available. It means that a complete set of information describing the effects of the close approach is obtained and it can be used by mission designers to help to reach the goals of the mission. In the simulations performed in the present research, the value used for this limit is half of the Sun-Earth distance. Regarding the mathematical model, the drag force is assumed to be proportional to the square of the velocity of the spacecraft with respect to the atmosphere and to the density of the atmosphere, which is obtained first from an exponential model relating altitude and density, and then from the more accurate ARDC 1959, a statistical model based in data obtained from sounding rockets and balloons experiments (Minzner et al. 1959) with density until $700 \mathrm{~km}$ of altitude (Zipfel 2007). The atmosphere is not assumed to be rotating. More complex models for the atmosphere are usually time dependent and, to obtain more general results, they were not used in this first study. Future research will consider those effects. The direction of the drag force is assumed to be opposite to the motion of the spacecraft. The lift force is assumed to have a similar form, proportional to the square of the velocity of the spacecraft with respect to the atmosphere and to the density of the atmosphere, but with the direction perpendicular to the motion of the spacecraft, with the positive sense pointing away from the Earth. Then, the goal of the present research is to map trajectories with respect to the variations of energy due to the close approaches under different initial conditions. The drag and the lift forces will be varied by changing the drag and lift coefficients, as explained in the next session.

\section{Definition of the problem and mathematical model}

The complete maneuver starts with the spacecraft coming from an elliptic or hyperbolic orbit around the Sun, in an initial position that can be considered far from the Earth. The incoming approach to the Earth causes the entrance of the spacecraft inside its atmosphere, 


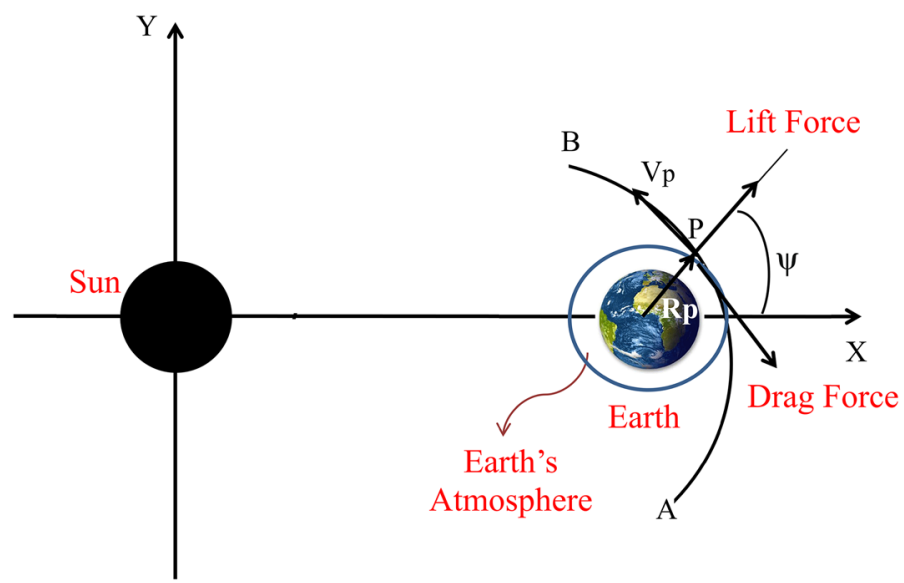

Fig. 1 Geometry of the close approach passing by the atmosphere of the Earth. $\psi$ is the angle of approach, which is the angle between the periapsis line and the Sun-Earth line

so the motion of the spacecraft becomes governed by the forces coming from the gravity of the Earth and the Sun and the atmospheric forces of lift and drag. The Earth is assumed to be in a circular orbit around the center of mass of the Sun-Earth system. Figure 1 shows the maneuver. The spacecraft approaches the Earth coming from point A, gets closer to the Earth, enters its atmosphere, completes the close approach and then goes to point $\mathrm{B}$. The point $\mathrm{P}$ is the perigee of the trajectory of the spacecraft around the Earth. The circle around the center of the Earth represents the atmosphere. When the spacecraft is at the point A or B, it is considered to be far enough from the Earth, so its motion can be considered keplerian around the Sun.

The equations of motion, in the rotating frame, are shown below (Eqs. 1-4). The rotating frame is a system of reference that rotates with the Sun-Earth line. In the present research it is also used the canonical system of units. Therefore, the Sun-Earth distance is defined as one unit of distance; the total mass of the Sun and the Earth is defined as the unit of mass and the unit of time is defined such that the period of motion of the Sun-Earth line is $2 \pi$. The equations of motion are the ones given by the circular restricted three-body problem combined with the atmosphere, opposite to the more used "Patched conics approach", and they are given by Szebehely (1967):

$$
\begin{aligned}
& \ddot{X}-2 \dot{Y}=\frac{\partial \Omega}{\partial X}+F_{X} \\
& \ddot{Y}+2 \dot{X}=\frac{\partial \Omega}{\partial Y}+F_{Y},
\end{aligned}
$$

where $\omega$ is the potential, given by

$$
\Omega=\frac{1}{2}\left(x^{2}+y^{2}\right)+\frac{(1-\mu)}{r_{1}}+\frac{\mu}{r_{2}}
$$

and $x, y$ are the coordinates of the spacecraft; $r_{1}$ and $r_{2}$ are the distances from the spacecraft to the Sun and the Earth, respectively; $\mu$ is the dimensionless mass of the Earth; $F_{x}$ and $F_{y}$ are the components of the force $(\vec{F})$ that comes from the atmosphere (drag and lift), which is the difference from the model given by Szebehely (1967). They are given by Guedes (1997), Schulz et al. (2002): 


$$
\vec{F}=\left(F_{X}, F_{Y}\right)=-C_{B} \rho V \vec{V}-C_{L B} \rho V^{2} \vec{S},
$$

where $C_{\mathrm{B}}=\frac{C_{\mathrm{D}} A}{2 m}$ is the ballistic coefficient, $C_{\mathrm{D}}$ is the drag coefficient (a parameter that takes into account the shape of the spacecraft); $C_{\mathrm{LB}}$ is the lift ballistic coefficient $\left(C_{\mathrm{LB}}=\frac{C_{\mathrm{L}} A}{2 m}\right.$, with $C_{\mathrm{L}}$ the lift coefficient); $A$ is the cross section area of the spacecraft; $\vec{V}$ is the velocity of the spacecraft with respect to the atmosphere, and $\vec{S}$ is the direction perpendicular to $\vec{V}$, pointing in the direction opposite to the Earth; $m$ is the mass of the spacecraft and $\rho$ is the density of the atmosphere, which is first estimated based on the exponential function shown below (Eq. 5):

$$
\rho=\rho_{0} \mathrm{e}^{\frac{-\left(h-h_{0}\right)}{H}}
$$

In Eq. (5) $\rho_{0}$ represents the density of the atmosphere in a given altitude (sea level in the present research) $h_{0} ; h$ is the altitude of the spacecraft and $H$ is the scale height, the vertical distance over which the density and pressure fall by a factor of 1/e. The values used in the present research are $1.225 \mathrm{~kg} / \mathrm{m}^{3}$ (NASA/NOAA 1976; Cardoso and Souza 2009) for $\rho_{0}$ and $6.5 \mathrm{~km}$ for $H$ (Reagan and Anandakrishnan 1993; Tewari 2007. Cardoso and Souza 2009). This is a simplified model and a second more accurate one is also used, which is the ARDC 1959 mentioned before).

\section{Specifying a close approach trajectory}

There are many choices to define a close approach trajectory, but the following set of variables are used here:

(a) $\psi$, the angle between the Sun-Earth line and the perigee line of the trajectory of the spacecraft around the Earth, usually called "angle of approach";

(b) $v_{\mathrm{p}}$, the velocity of the spacecraft when passing by the perigee;

(c) $h_{\mathrm{p}}$, the altitude of the perigee of the orbit of the spacecraft around the Earth.

After defining those variables, it is possible to make an algorithm to map the trajectories. It is shown below and it can measure the effects of the drag and lift forces in the trajectories of the spacecraft passing by the atmosphere of the Earth.

(i) The first step is to perform a numerical integration in negative times, with the initial conditions of the spacecraft at the perigee, to measure the parameters of the orbit before the close approach. This numerical integration ends when the spacecraft reaches the point A, as shown in Fig. 1, which is a point where the distance from the Earth is equal to half of the Sun-Earth distance. This is a point that can be considered far enough from the Earth, such that the two-body dynamics are valid to obtain the Sun-spacecraft energy before the close approach. Several tests were made to verify if the backward integration works well in this system, and the results showed that this technique is valid in the present situation;

(ii) After that, another numerical integration is performed, again with the initial conditions of the spacecraft at the perigee, but now for positive times, to verify the status of the orbit after the close approach. The numerical integration now ends when the spacecraft collides with the Earth or reaches the point B, also shown in Fig. 1. This point B is also assumed to be far enough from the Earth, so the system Sun-spacecraft is one more time keplerian. The same distance of half of the Sun-Earth distance is used to define this point. Then, the two-body energy Sun-spacecraft is calculated again, this time after the maneuver. 
Therefore, using this sequence of steps for a large range of values for the ballistic coefficient and other parameters that specify the close approach trajectories, it is possible to understand the effects of the drag and the lift forces in the trajectory of the spacecraft. The numerical integrations in the present paper were made using a Runge-Kutta integrator of fourth order and step size control, with a numerical accuracy of $10^{-8}$.

\section{Results}

The results of the simulations made here can be seen in figures that give the energy variation per unit of mass due to the passage of the spacecraft by the atmosphere of the Earth (in canonical units), in the vertical axis, as a function of the ballistic coefficient (in $\mathrm{km}^{2} / \mathrm{kg}$ ) in the horizontal axis. The idea of using this form to show the results is that the best values for the ballistic coefficients can be found for a specific mission and then the combination of spacecraft shape, area, mass and trajectory can be made to achieve this value. The value used for the perigee velocity is 1.0 canonical units, which happened to be a good value to show the main characteristics of the phenomenon. Other values were used, with similar results, so they are not shown here. It is important to mention that 1.0 canonical unit is equivalent to $29.78 \mathrm{~km} / \mathrm{s}$, the orbital velocity of the Earth around the Sun. Numerical preliminary tests showed that values well below 1.0 canonical units reduce the number of captures by the Earth and values well above this limit lead to close approaches that have very little effects on the trajectory of the spacecraft. Regarding the angle of approach $(\psi)$, three values were used: $90^{\circ}, 180^{\circ}$ and $270^{\circ}$. The reason for choosing those values is that they represent the particular cases of maximum gain of energy $\left(270^{\circ}\right)$, maximum loss of energy $\left(90^{\circ}\right)$ and zero variation of energy $\left(180^{\circ}\right)$. This dependency comes from the fact that the velocity of the spacecraft before and after the maneuver is a function of this variable, as seen in detail in Broucke (1988). The search for maximum variations of energy are common in the literature, as mentioned before in the missions that use Mars and/or Venus to reach the exterior planets, but the search for trajectories that have energy loses is a new aspect of the present research. This case has important applications when sending a spacecraft to the interior of the Solar System or when studying a return mission to the Earth, as mentioned before. Figures 2, 3, 4, $5,6,7,8,9$ and 10 show the main results of the combined effects of drag and lift. In those figures, $L / D$ represents the ratio of the forces lift/drag. This term was chosen to specify the magnitude of the lift force, so it is necessary to remember that the lift force is obtained multiplying $L / D$ by the drag force.

Another important point is related to which values for the ballistic coefficient should be used. According to the literature, one of the definitions of the ballistic coefficient is the relation between the spacecraft mass $(m)$ and the product of the perpendicular flow area $(A)$ multiplied by the drag coefficient $\left(C_{\mathrm{D}}\right)$, which means $\left(m / A C_{\mathrm{D}}\right)$. The reentry capsules can have values above $300 \mathrm{~kg} / \mathrm{m}^{2}$ (Davies and Arcadi 2006), and the more used range is from 10 to $200 \mathrm{~kg} / \mathrm{m}^{2}$ for traditional satellites. In this paper, the inverse relation $\left(A C_{\mathrm{D}} / \mathrm{m}\right)$, also defined as ballistic coefficient, and represented by the symbol $C_{\mathrm{B}}$, is used. This means that the traditional range should be $C_{\mathrm{B}}>1.0 \times 10^{-3} \mathrm{~m}^{2} / \mathrm{kg}$ (Kuga et al. 2011). So, in this study, the range of values of $C_{\mathrm{B}}$ goes from zero, which means that there are no effects of the atmosphere on the motion of the spacecraft $\left(C_{\mathrm{D}}=0\right.$ or the density of the atmosphere is negligible at the altitude considered) until $10 \times 10^{-6} \mathrm{~km}^{2} / \mathrm{kg}$. This later value represents a spacecraft with an area of the order of $1000 \mathrm{~m}^{2}$, considering a mass of $100 \mathrm{~kg}$ and assuming $C_{\mathrm{D}}=2$. Areas as large as this one can be obtained with the use of a large panel in the spacecraft. Although 


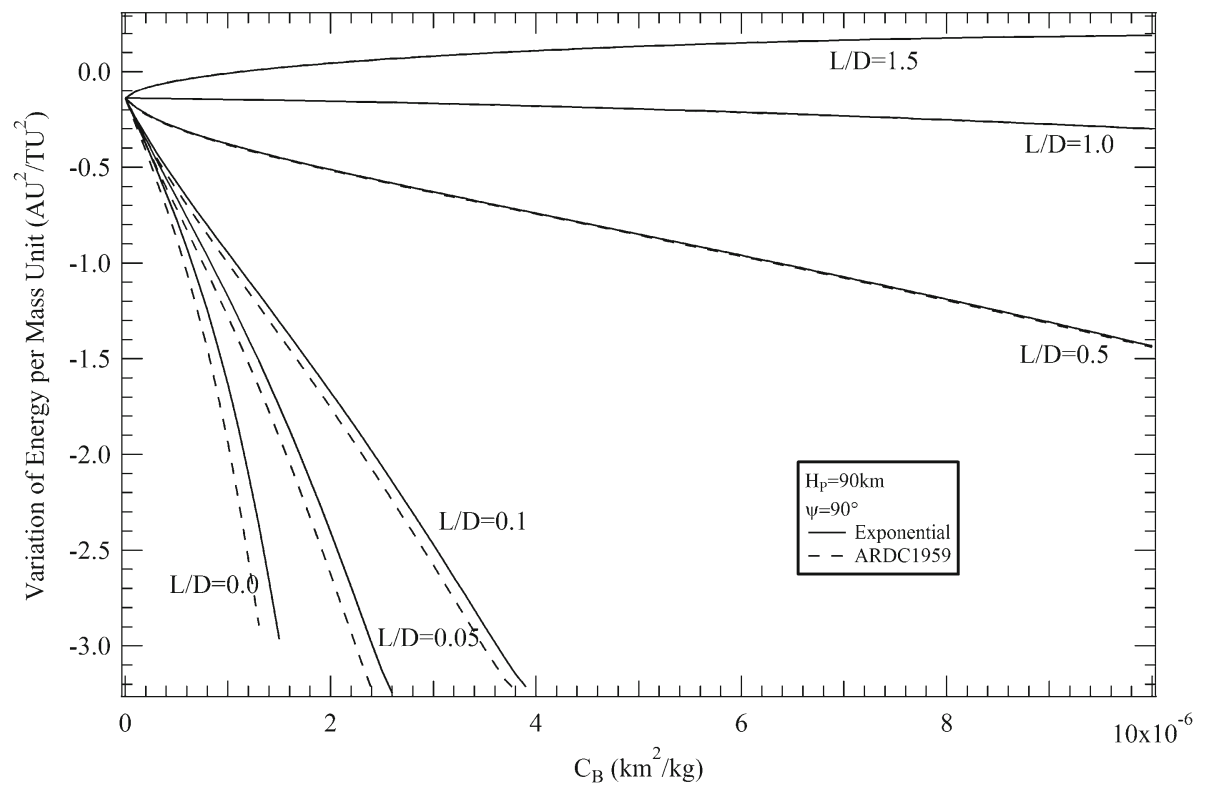

Fig. 2 Variation of energy per unit of mass due to the close approach (canonical units) as a function of the ballistic coefficient $\left(\mathrm{km}^{2} / \mathrm{kg}\right)$ until $10 \times 10^{-6}$ for $h_{\mathrm{p}}=90 \mathrm{~km}$ and $\psi=90^{\circ}$, for both atmospheric models considered, for $L / D=0.00,0.05,0.10,0.50,1.00$ and 1.50

some of those values are not practical nowadays, they show theoretical possibilities that may be of interest in the future. In order to make a more general study, the same plots are made for shorter intervals of the ballistic coefficients, from 0.0 until $1 \times 10^{-6} \mathrm{~km}^{2} / \mathrm{kg}$ and then from 0.0 to $0.5 \times 10^{-6} \mathrm{~km}^{2} / \mathrm{kg}$, to see better some details of the region with more practical applications. The range of values for the drag coefficient is from 2.0 to 2.3, which depends on the interaction between the surface of the spacecraft and the atmosphere (Montenbruck and Gill 2000). The relationship between the lift and drag forces $(L / D)$, proportional to the coefficients $\left(C_{\mathrm{L}} / C_{\mathrm{D}}\right)$, is known by aerodynamic efficient ratio and it is a function of the Mach number, the angle of attack, the flow molecular number and other variables, like the shape of the spacecraft. For winged Glider (like the orbiter space shuttle) configuration it is possible to find $L / D<1.5$ (Armellin et al. 2007). If the angle of attack has negative value it is possible that $L / D<0.0$, but this case is not considered in the present paper. For traditional spacecraft or satellite bodies, the usual ratio can be between $0<L / D<1.0$, where the principal aerodynamic force acting in the spacecraft is the drag (Sims et al. 2000; Lewis and McRonald 1992). In order to cover a larger interval to understand the effects of this parameter, the values $0.00,0.05,0.10,0.50,1.00$ and 1.50 were used for this lift/drag ratio.

The results show some important characteristics of this type of maneuver. Some of those properties are quantification of expected results, but this is also important in this problem. The first fact verified is that when $\psi=90^{\circ}$ there is a negative variation of energy for lower values of the ballistic coefficient and, when $\psi=270^{\circ}$, there is a positive variation of energy for the same lower values of the ballistic coefficient. The situation where $\psi=$ $180^{\circ}$ represents the case where there is no variation of energy due to the gravitational part of the maneuver. This analysis is made based in the initial points of the plots $\left(C_{\mathrm{B}}=0\right)$, 


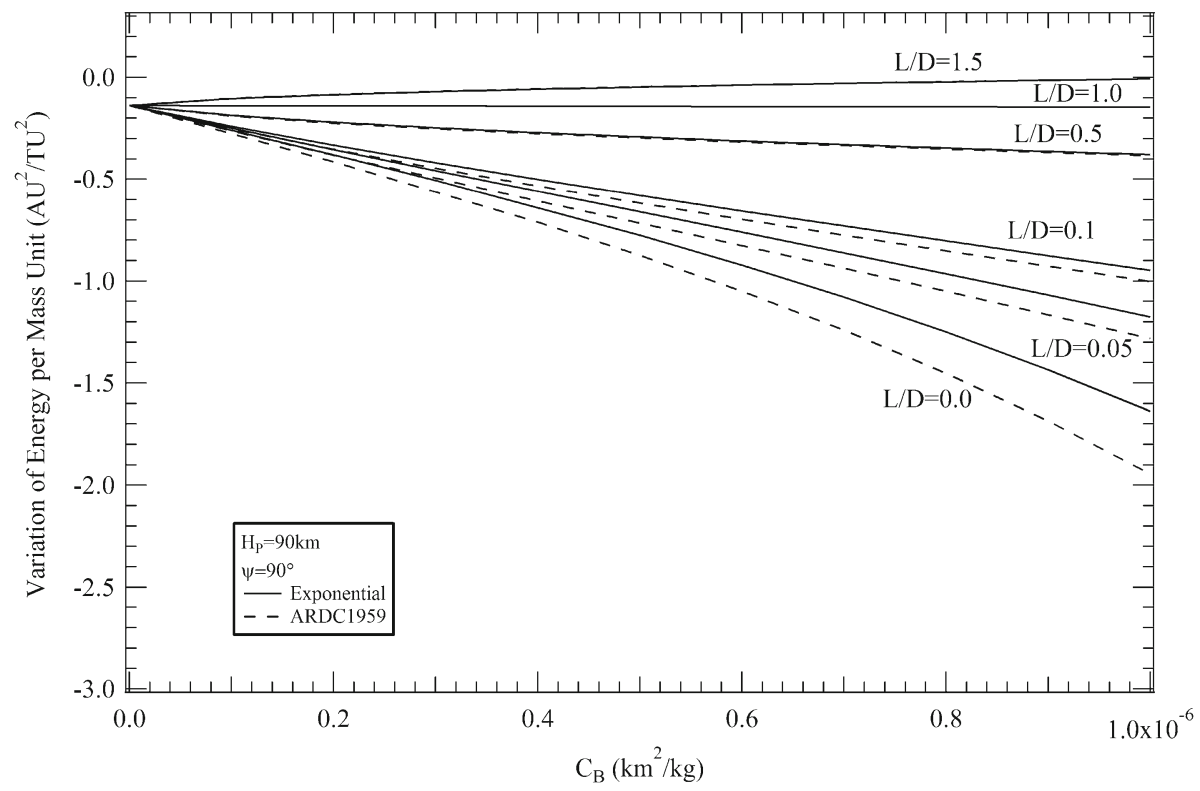

Fig. 3 Variation of energy per unit of mass due to the close approach (canonical units) as a function of the ballistic coefficient $\left(\mathrm{km}^{2} / \mathrm{kg}\right)$ until $1.0 \times 10^{-6}$ for $h_{\mathrm{p}}=90 \mathrm{~km}$ and $\psi=90^{\circ}$, for both atmospheric models considered, for $L / D=0.00,0.05,0.10,0.50,1.00$ and 1.50

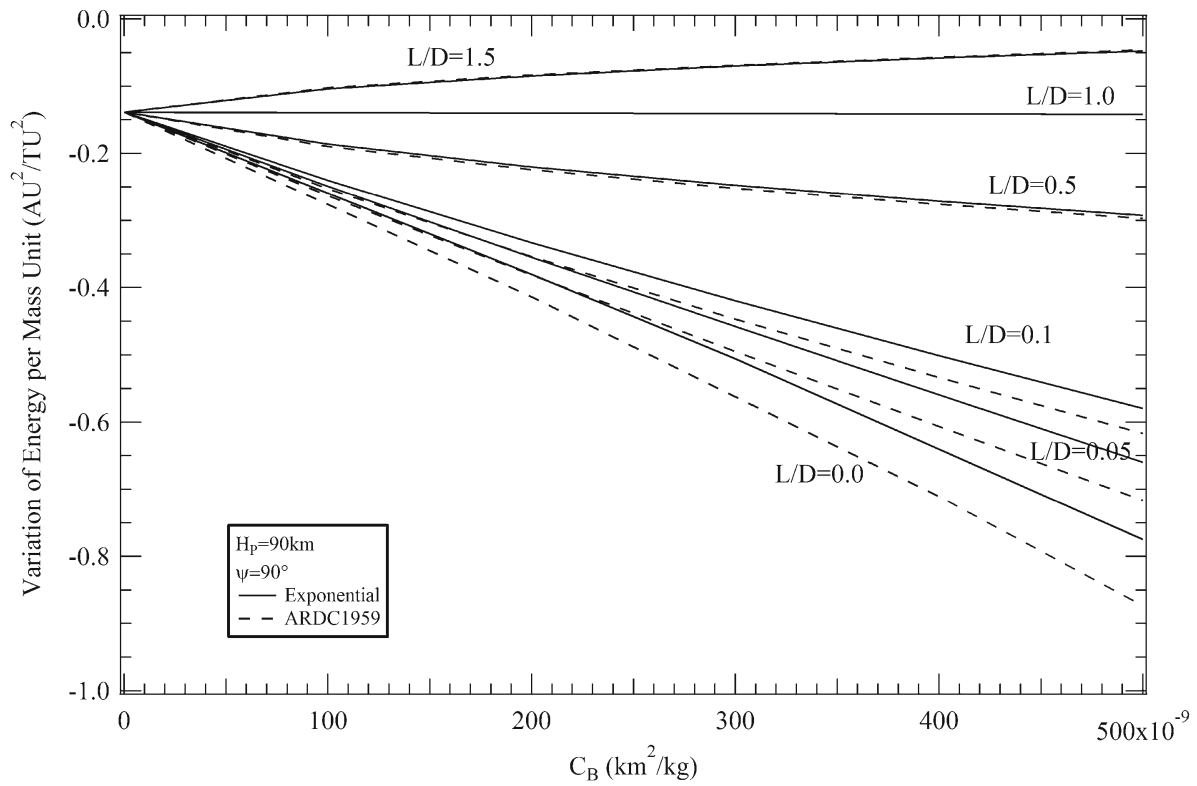

Fig. 4 Variation of energy per unit of mass due to the close approach (canonical units) as a function of the ballistic coefficient $\left(\mathrm{km}^{2} / \mathrm{kg}\right)$ until $500 \times 10^{-9}$ for $h_{\mathrm{p}}=90 \mathrm{~km}$ and $\psi=90^{\circ}$, for both atmospheric models considered, for $L / D=0.00,0.05,0.10,0.50,1.00$ and 1.50 


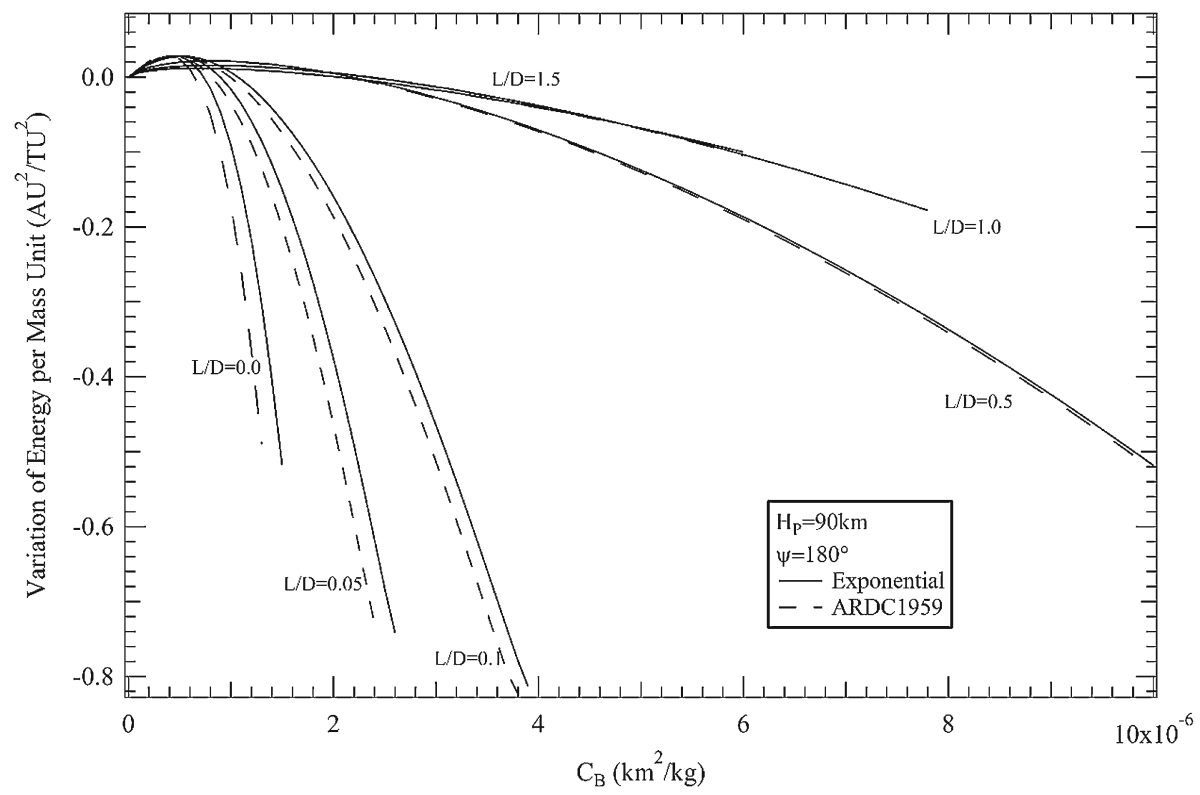

Fig. 5 Variation of energy per unit of mass due to the close approach (canonical units) as a function of the ballistic coefficient $\left(\mathrm{km}^{2} / \mathrm{kg}\right)$ until $10 \times 10^{-6}$ for $h_{\mathrm{p}}=90 \mathrm{~km}$ and $\psi=180^{\circ}$, for both atmospheric models considered, for $L / D=0.00,0.05,0.10,0.50,1.00$ and 1.50

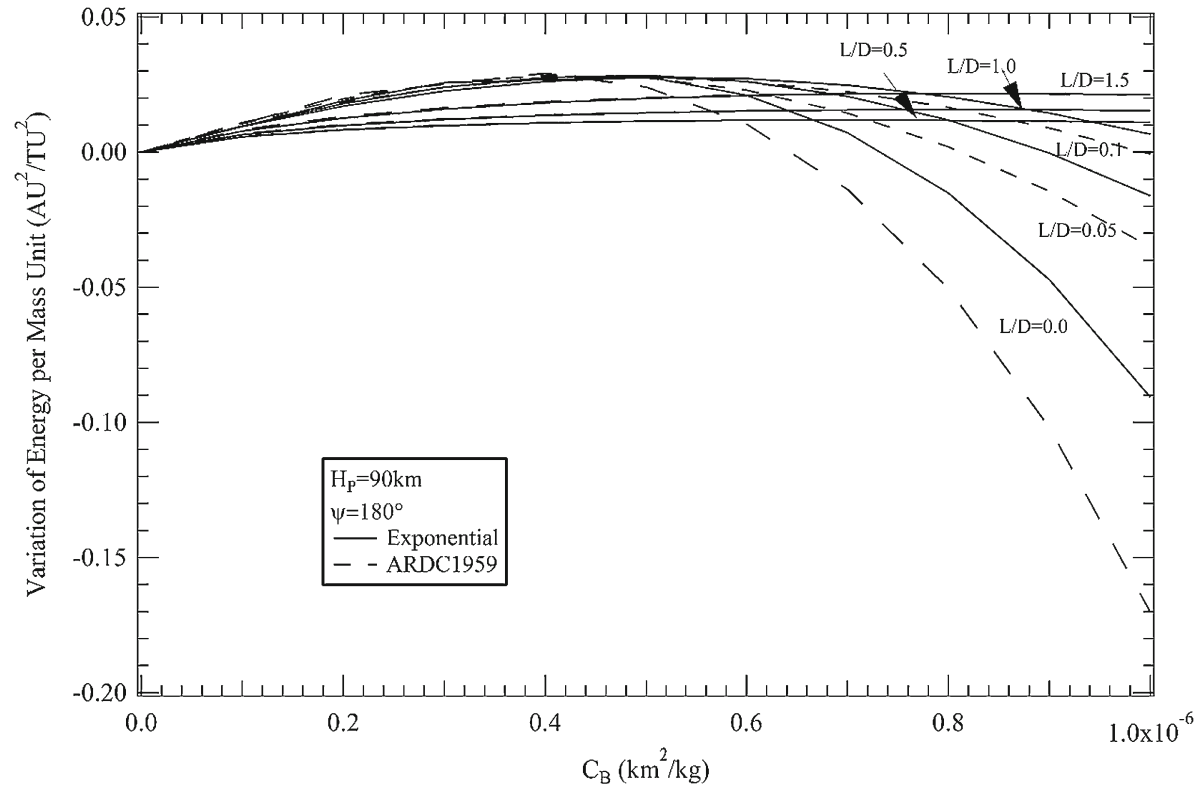

Fig. 6 Variation of energy per unit of mass due to the close approach (canonical units) as a function of the ballistic coefficient $\left(\mathrm{km}^{2} / \mathrm{kg}\right)$ until $1.0 \times 10^{-6}$ for $h_{\mathrm{p}}=90 \mathrm{~km}$ and $\psi=180^{\circ}$, for both atmospheric models considered, for $L / D=0.00,0.05,0.10,0.50,1.00$ and 1.50 


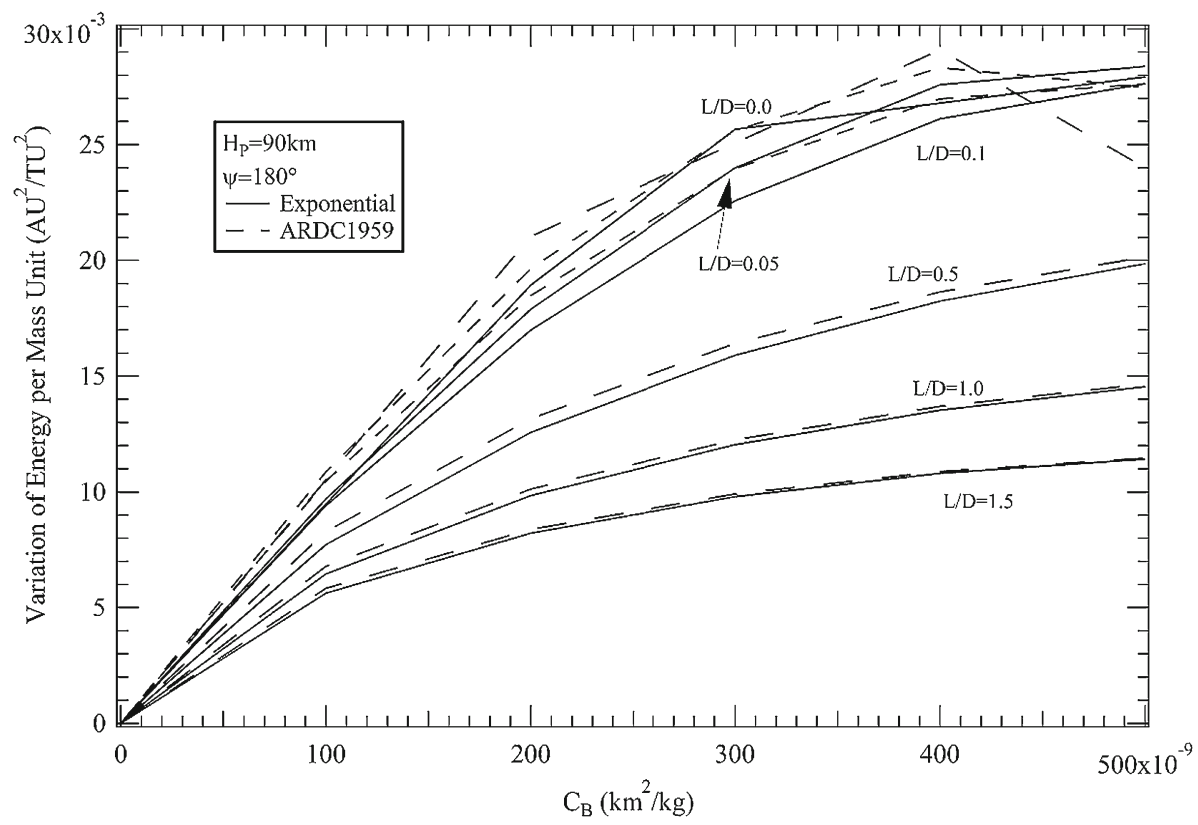

Fig. 7 Variation of energy per unit of mass due to the close approach (canonical units) as a function of the ballistic coefficient $\left(\mathrm{km}^{2} / \mathrm{kg}\right)$ until $500 \times 10^{-9}$ for $h_{\mathrm{p}}=90 \mathrm{~km}$ and $\psi=180^{\circ}$, for both atmospheric models considered, for $L / D=0.00,0.05,0.10,0.50,1.00$ and 1.50

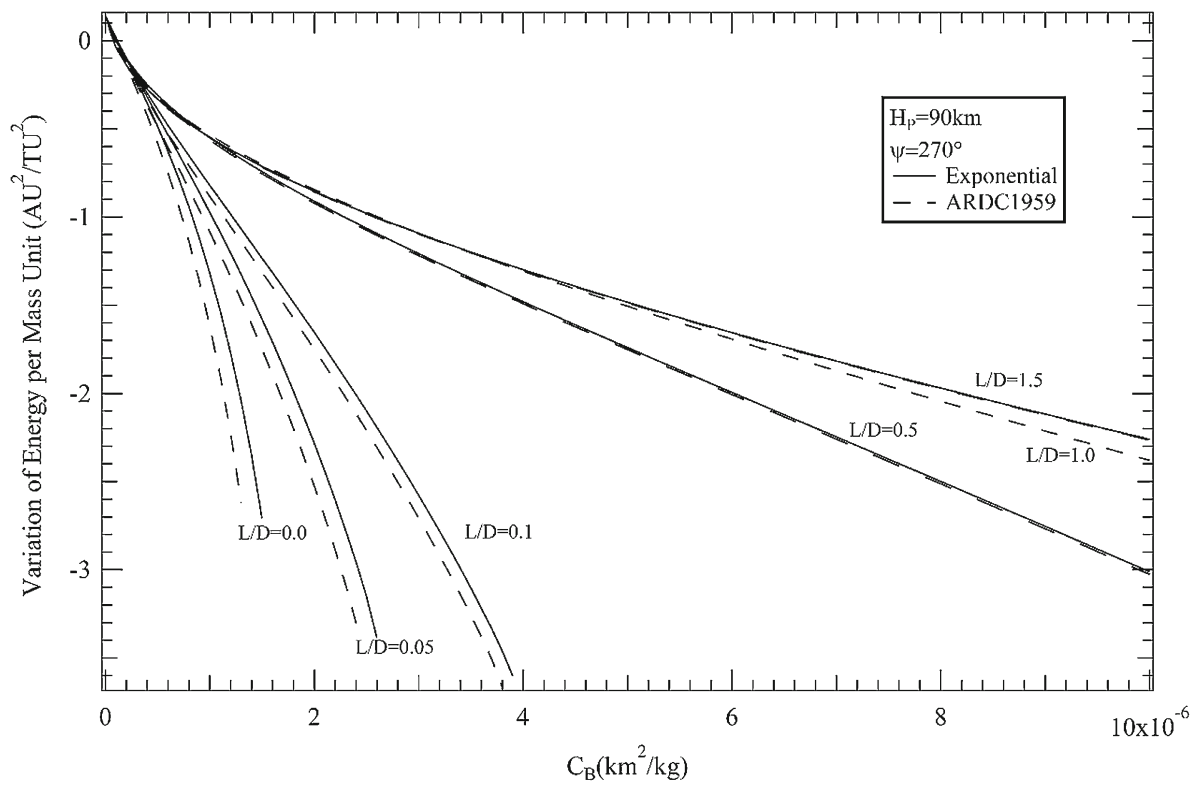

Fig. 8 Variation of energy per unit of mass due to the close approach (canonical units) as a function of the ballistic coefficient $\left(\mathrm{km}^{2} / \mathrm{kg}\right)$ until $10 \times 10^{-6}$ for $h_{\mathrm{p}}=90 \mathrm{~km}$ and $\psi=270^{\circ}$, for both atmospheric models considered, for $L / D=0.00,0.05,0.10,0.50,1.00$ and 1.50 


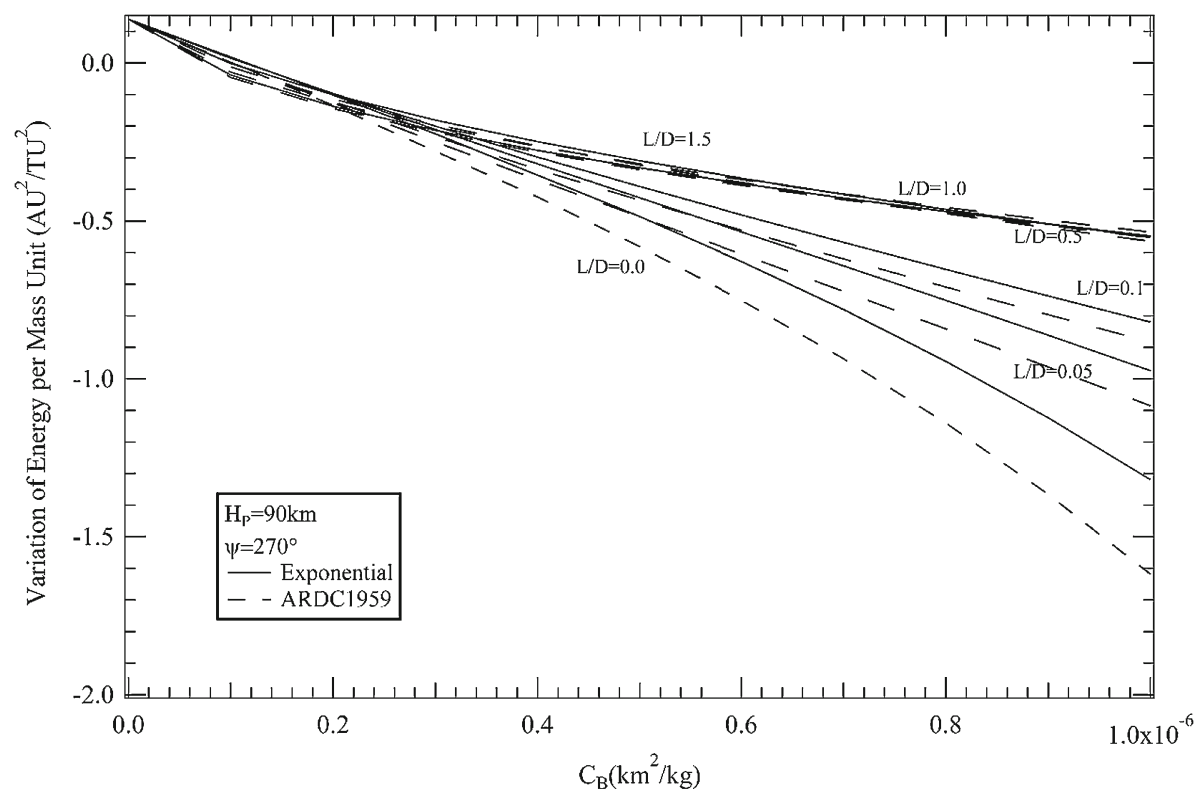

Fig. 9 Variation of energy per unit of mass due to the close approach (canonical units) as a function of the ballistic coefficient $\left(\mathrm{km}^{2} / \mathrm{kg}\right)$ until $1.0 \times 10^{-6}$ for $h_{\mathrm{p}}=90 \mathrm{~km}$ and $\psi=270^{\circ}$, for both atmospheric models considered, for $L / D=0.00,0.05,0.10,0.50,1.00$ and 1.50

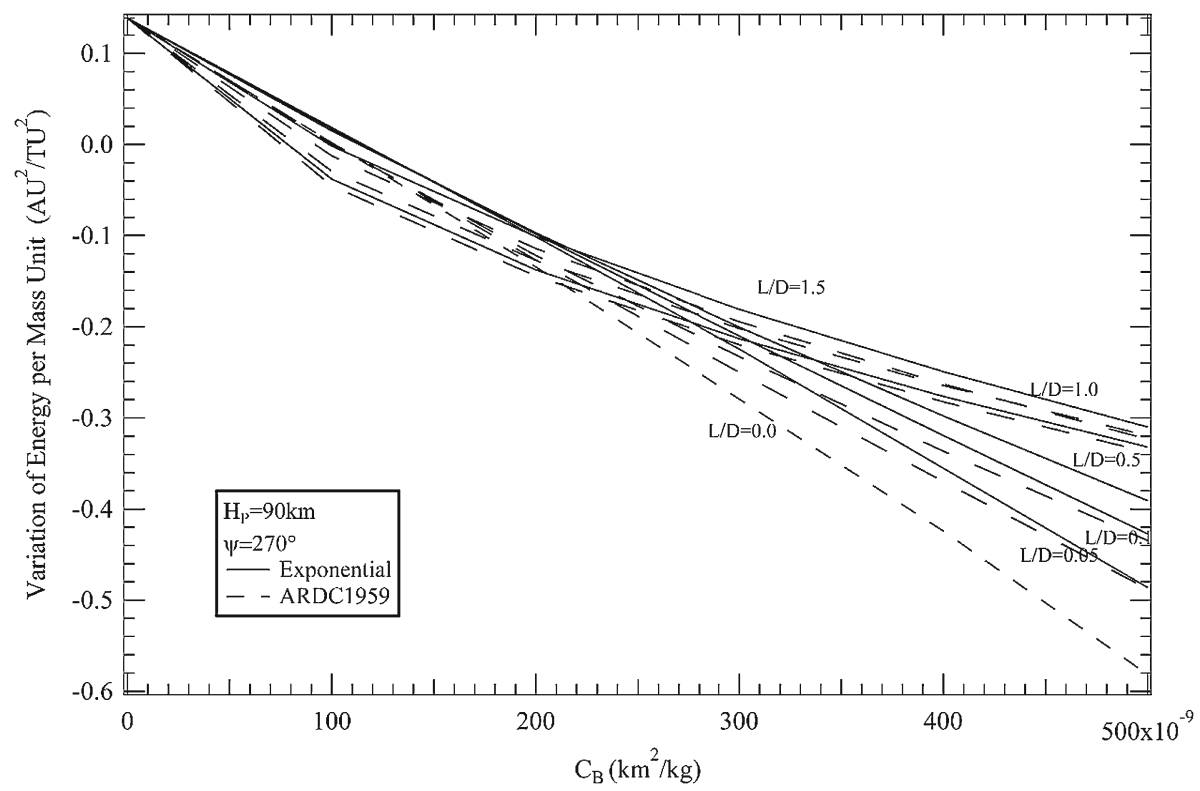

Fig. 10 Variation of energy per unit of mass due to the close approach (canonical units) as a function of the ballistic coefficient $\left(\mathrm{km}^{2} / \mathrm{kg}\right)$ until $500 \times 10^{-9}$ for $h_{\mathrm{p}}=90 \mathrm{~km}$ and $\psi=270^{\circ}$, for both atmospheric models considered, for $L / D=0.00,0.05,0.10,0.50,1.00$ and 1.50 
which represents the maneuvers without the effects of the atmosphere (the vertical axis). This fact is well explained by Broucke (1988), but it is verified and quantified in the present research under the "Restricted Three Body Problem", which is a better model compared to the "patched-conics" approach, which is the model used in Broucke (1988). When the atmosphere starts to be present, which means moving away from the vertical axis of the figures, there are important modifications in those maneuvers, as described later in the present paper.

Besides the quantification of the above cited expected results, some other facts are observed by looking at the results of the present research. Those facts are related to the presence of the atmosphere. The first one is that the use of lift, which means that there is a force applied in the direction opposite to the Earth, making the trajectory of the spacecraft to have a smaller angle of curvature. In this situation the variations of energy are decreased, because the gravity part of the maneuver is proportional to this variable. It is also noted that, as expected, increasing the value of the lift amplifies those effects. These results can quantify very well those aspects in the figures shown before. It is important to note here that there are two forms to increase the lift force. The first one is by moving away from the vertical axis, because it increases the value of the ballistic coefficient and the lift force is defined as a function of the drag force. The second form is to increase the value of $L / D$, which is done by moving from one line to the other, in the figures shown here.

\subsection{The situation where the angle of approach is $90^{\circ}$}

Figures 2, 3 and 4 are made for situations where the angle of approach is $90^{\circ}$, so there are energy losses due to the gravitational part of the maneuver. In those maneuvers, when the lift is not too strong ( $L / D$ below 1.0), there is always an increase in the variation of energy with the value of the ballistic coefficient, because the drag always reduces the energy of the spacecraft, so amplifying the effects of the gravity part of the maneuver. Besides this direct source of removal of energy, the drag also reduces the velocity of the spacecraft, so increasing the turning angle of the maneuver, and then removing more energy from the spacecraft due to the gravitational part of the maneuver. This increase in the reduction of energy is very dependent on the value of the ballistic coefficient and the final results of the maneuver are much larger when compared to the pure gravitational maneuver. It is noted that the increase of the lift force reduces the angle of curvature, due to its component in the direction opposite to the Earth, so it reduces the effects of the gravitational part of the maneuver. A near-equilibrium situation occurs when $L / D=1.0$. The line representing the variation of energy in this case is almost horizontal, which means that the losses due to the increase of the drag removal of energy are compensated by the fact that the lift force is decreasing the angle of curvature of the trajectory, so the gravitational part of the maneuver is less effective in taking energy from the spacecraft. This equilibrium situation makes the variation of energy to be independent of the ballistic coefficient. For values of lift/drag larger than 1.0, the loss of energy is decreased until a point of equilibrium, which is near $C_{\mathrm{B}}=1.0 \times 10^{-6} \mathrm{~km}^{2} / \mathrm{kg}$, at the extreme right of Fig. 3. This is a point with zero variation of energy, because the resulting trajectory inverted the sense of motion and now gives energy to the spacecraft, in a amount equivalent to the loss due to the drag force. After this point the net result is positive, with the gains being larger than the losses of energy, and the final result is a maneuver that increase the energy of the spacecraft. Another observation that can be made is that the exponential model is very good in the situations where $L / D>0.5$, and it loses quality for the lower values of this ratio. 


\subsection{The situation where the angle of approach is $180^{\circ}$}

In the cases shown in Figs. 5, 6 and 7 the angle of approach is $180^{\circ}$, so there are no energy variation due to the gravitational part of the maneuver. The effect of the atmosphere is to generate a positive variation of energy at the beginning of the curves (for $C_{\mathrm{B}}$ up to around $5.0 \times 10^{-6} \mathrm{~km}^{2} / \mathrm{kg}$ ), because the loss of energy coming from the drag force is smaller than the gains that are generated by the variation of the geometry caused by the presence of the atmosphere. A maximum positive value for this variation is obtained during the sequence of values used. After this maximum value the variation of energy starts to decrease, reaching a zero value again for a specific value of the ballistic coefficient that depends on the $L / D$ ratio. At this point the variation of energy is zero again. After this point the losses are larger than the gains, and the net result is a negative variation of energy. The increase of the lift force reduces the effects of the maneuver, as noted in the previous cases, because the angle of curvature is less modified by the atmosphere. The exponential model is a good approximation for higher values of $L / D$ cases, as in the previous situations. The final result of the atmosphere is that, in a situation where the variation of energy should be zero, it can have positive and negative values.

\subsection{The situation where the angle of approach is $270^{\circ}$}

In other cases (Figs. 8, 9, 10), in situations where the angle of approach is $270^{\circ}$ (so there are energy gains due to the gravitational part of the maneuver), the effect of the atmosphere is to reduce the energy gains, because the drag contribution decreases the energy of the spacecraft. It is noted that the magnitudes of the variations of energy are strongly increased by the presence of the atmosphere. So, this is an interesting situations where a maximum gain in energy is expected, but large negative results can be obtained. Once again the increase of the lift force reduces the strong effects of the atmosphere.

\subsection{General comments}

From the collection of results shown here, it is also verified that when the drag and lift coefficients are above a certain limit, the atmosphere dominates the motion of the spacecraft with respect to the direction of motion, and the curvatures of the trajectories can be in the opposite directions, compared to the ones expected from the pure gravitational maneuver. It generates situations where there are losses of energy where gains were expected, based only in the angle of approach analysis, and vice-versa. Figure 2 shows a good example of this situation. The maneuver is made with an angle of approach of $90^{\circ}$, so a pure gravitational maneuver would give the maximum loss of energy. The drag force also removes energy from the spacecraft, so it works in the same direction of the gravity forces. The line of $L / D=1.5$ shows a situation where the variation of energy increases with the ballistic coefficients after a certain value of this coefficient, reaching a positive value for $C_{\mathrm{B}}=1.0 \times 10^{-6} \mathrm{~km}^{2} / \mathrm{kg}$. But, it is necessary to consider that the lift force pushes the spacecraft away from the Earth, so decreasing the angle of curvature of the trajectories and, consequently, decreasing the effects of the gravitational part of the maneuver. After some point, it changes the direction of the motion, as shown in Fig. 11, which causes the change of the sign of the energy variation. Physically, it means that the energy variation can be increased or decreased by the atmosphere. It should be noted that this manuscript did not consider the rotation of the atmosphere or ascending currents.

To obtain the zero variation of energy situations, the lift force modified the trajectory of the spacecraft in such way that the velocity of the spacecraft rotates in the clockwise direction. 


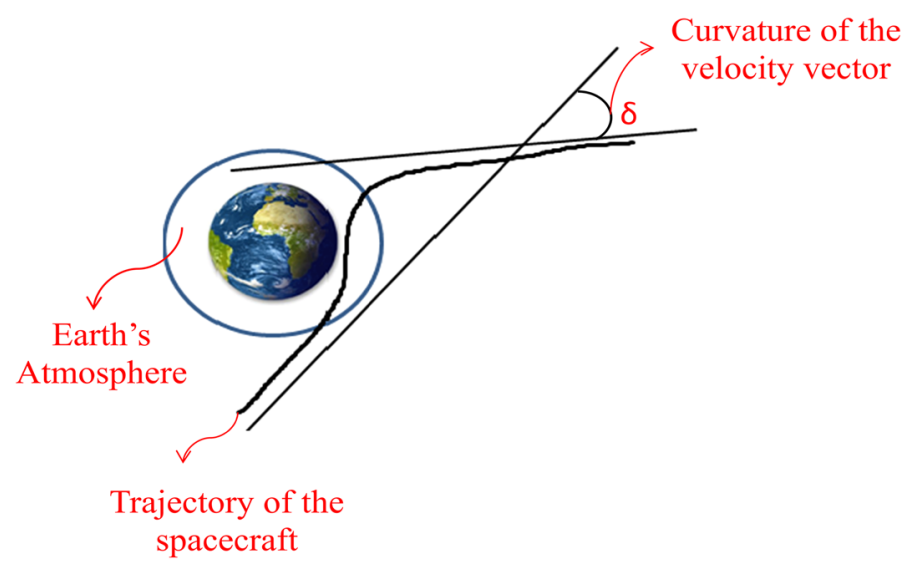

Fig. 11 Trajectory of the spacecraft considering lift and drag

So, in this particular point, energy losses and gains are the same and the final result is a maneuver that changes the direction of the velocity vector of the spacecraft with respect to the Sun, but that does not modify the magnitude of that velocity, so not giving variation of energy. This is a new alternative for close approach trajectories, if the goal is to change the direction of the trajectory without the use of propulsion, but keeping the energy constant. It means to make a modification of the eccentricity of the orbit of the spacecraft around the Sun, but keeping the value of the semi-major axis constant. A maneuver like that can be used in missions that have the goal of passing close to more than one celestial body, like finding an elliptical trajectory around the Sun that has an apoapsis near the orbit of the Earth and a periapsis near the orbit of Venus, such that the spacecraft can pass near both bodies. After this point, the gains of energy are larger than the losses and the net result is a gain of energy. Those figures also show that there are situations of another type of equilibrium, where the variation of energy is independent of the magnitude of the drag and lift forces, if their ratio remains constant. The physical reason is that under this specific ratio $(L / D=1.0$ in Fig. 2) the energy losses produced by the drag force are compensated by the reduction of the losses due to the gravity part of the maneuver, obtained thanks to the decrease of the angle of curvature made by the lift force, with a consequent reduction in the effects of the close approach. This fact was noted due to the low values of the lift/drag ratio, when compared to the literature, that always concentrated in high values for this ratio, reaching an order of 10.0 , because they give higher escape velocities.

In general, the results shown here confirm that there is an almost linear relation between the variations of energy and the ballistic coefficient most of the time, as also shown in Lewis and McRonald (1992), with few exceptions of this rule. It means that the presence of the lift does not change this linearity. This characteristic is important, because it makes easier the work of predicting variations of energy. Analytical equations based on the simulations shown here can easily be made.

\section{Conclusions}

A numerical algorithm to measure the effects of the drag and lift forces caused by the atmosphere in a close approach between the Earth and a spacecraft is developed and imple- 
mented. The results quantify the effects caused by the atmosphere in the trajectories of spacecrafts. In general, the drag force removes energy from the spacecraft, causing negative variations of energy, even in geometries where an increase of energy should occur due to the gravitational part of the maneuver. The lift force helps to change the curvature of the trajectory of the spacecraft around the Earth, changing the variation of energy due to the gravitational part of the maneuver. This fact opens several new characteristics of the trajectories, which were shown in detail.

The relation between the energy and the ballistic coefficients is almost linear in all cases studied when drag and lift are considered. Several interesting situations, like regions where the variation of energy is independent of the effects of the atmosphere due to the equilibrium of the effects that increase and decrease the energy of the spacecraft are found. There are also situations where there are reversions between gains and losses of energy depending on the effects of the atmosphere, and maneuvers with angle of approach of $90^{\circ}$ can generate gains of energy. Due to the same reason, maneuvers with angle of approach of $270^{\circ}$ can generate losses of energy. Maneuvers with angle of approach of $180^{\circ}$, that were expected to have a zero variation of energy, can have positive and negative values for the variation of energy. The present study can help to plan missions that have a passage near the Earth as one of the steps.

Acknowledgments The authors wish to express their appreciation for the support provided by Grants \#473387/2012-3, 473164/2013-2 and 304700/2009-6 from the National Council for Scientific and Technological Development (CNPq); Grants \# 2011/08171-3, 2011/13101-4, 2014/06688-7 and 2012/21023-6 from São Paulo Research Foundation (FAPESP) and the financial support from the National Council for the Improvement of Higher Education (CAPES).

\section{References}

Armellin R, Lavagna M, Starkey RP, Lewis MJ (2007) Aerogravity-assist maneuvers: coupled trajectory and vehicle shape optimization. J Spacecr Rockets 44(5):1051

Armellin R, Lavagna M, Ercoli-Finzi A (2006) Aero-gravity assist maneuvers: controlled dynamics modeling and optimization. Celest Mech Dyn Astron 95:391-405

Bonfiglio EP, Longuski JM, Vinh NX (2000) Automated design of Aerogravity-assist trajectories. J Spacecr Rockets 37(6):768

Broucke RA (1988) The celestial mechanics of gravity assist. AIAA paper 88-4220. In: AIAA/AAS astrodynamics conference, Minneapolis, MN, 15-17 Aug, 1988

Cardoso G, Souza M (2009) Análise e Simulação de Reentradas Atmosféricas Controladas (in Portuguese). INPE, São José dos Campos

Carvell R (1986) Ulysses-the sun from above and below. Space 1:18-55

D’Amario LA, Byrnes DV, Stanford RH (1982) Interplanetary trajectory optimization with application to Galileo. J Guid Cont Dyn 5(5):465-471

Davies C, Arcadi M (2006) Planetary mission entry vehicles quick reference guide, V3.0, NASA SP 2006-3401

Dunham D, Davis S (1985) Optimization of a multiple lunar- swing-by trajectory sequence. J Astron Sci 33(3):275-288

Elices T (1995) Maximum Delta-V in the aerogravity assist maneuver. J Spacecr Rockets 32(5):921-922

Flandro G (1966) Fast reconnaissance missions to the outer solar system utilizing energy derived from the gravitational field of jupiter. Astr Acta 12:329-337

Gillespie RW, Ross S (1967) Venus-swingby mission mode and its role in the manned exploration of Mars. J Spac Roc 4(2): 170

Gomes VM, Prado AFBA (2010) A study of the impact of the initial energy in a close approach of a cloud of particles. WSEAS Trans Math 9:811-820

Gomes VM, Prado AFBA, Golebiewska J (2013) Dynamics of space particles and spacecrafts passing by the atmosphere of the Earth. Sci World J 2013:1-6 
Guedes UTV (1997) Análise de dispersão da trajetória de reentrada em relação ao ponto de pouso, utilizando sistema geocêntrico inercial e manobras laterais, in Portuguese. Ph.D Dissertation, Institiuto Nacional de Pesquisas Espaciais, 1997

Helton AF, Strange NJ, Longuski JM (2002) Automated desing of the Europa orbiter tour. J Spac Roc 39(1): $17-22$

Hollister WM, Menning MD (1970) Periodic swing-by obits between Earth and Venus. J Spac Roc 7(10): 1193-1199

Ivanov PB, Papaloizou JCB (2011) Close encounters of a rotating star with planets in parabolic orbits of varying inclination and the formation of hot Jupiters. Celest Mech Dyn Astron 111:51-82

Kawaguchi J, Yamakawa H, Uesugi T, Matsuo H (1995) On making use of lunar and solar gravity assists in LUNAR-A PLANET-b missions. Acta Astron 35(Suppl):633-646

Kuga H, Carrara V, Rao K (2011) Satélites artificiais-movimento orbital (in Portuguese). INPE, São José dos Campos

Laskar J, Marchal C (1984) Triple close approach in the three body problem: a limit for the bounded orbits. Celest Mech 32:15-28

Lavagna M, Povoleri A, Finzi AE (2005) Interplanetary mission design with aero-assisted manoeuvres multiobjective evolutive optimization. Acta Astron 57:498

Lewis MJ, McRonald AD (1992) Design of hypersonic waveridders for aeroassisted interplanetary trajectories. J Spacecr Rockets 29(5):653

Lohar FA (1996) Optimal aero-gravity assist with heliocentric plane change. Acta Astron 38(4-8):445

Lohar FA, Mateescu D, Misra AK (1994) Optimal atmospheric trajectory for aero-gravity-assist. Acta Astrona 32(2):89

Lohar FA, Misra AK, Mateescu D (1997) Mars-jupiter aerogravity assist trajectories for high-energy missions. J Spacecr Rockets 34(1):16

Longuski JM, Williams SN (1995) The last grand tour opportunity to Pluto. J Astron Sci 39(1991):359-365

Marsh SM, Howell KC (1988) Double lunar swing by trajectory design. AIAA paper 88-4289

McConaghy TT, Debban TJ, Petropoulos AE, Longuski JM (2003) Design and optimization of low-thrust gravity trajectories with gravity assist. J Spacecr Rockets 40(3):380-387

McRonald AD, Randolph JE (1992) Hypersonic maneuvering for augmenting planetary gravity assist. J Spacecr Rockets 29(2):216

Minovich MA (1961) A method for determining interplanetary free-fall reconnaissance trajectories. JPL Tech Memo 312-130. Pasadena: JPL

Minzner R, Champion K, Pond H (1959) The ARDC model atmosphere, 1959. Air Force Surveys in Geophysics N115

Montenbruck O, Gill E (2000) Satellite orbits—models, methods, and applications. Springer, Berlin

NASA/NOAA (1976) US standard atmosphere 1976. NASA TM-X74335, Washington DC

Okutsu M, Yam CH, Longuski JM (2006) Low-thrust trajectories to jupiter via gravity assists from Venus, Earth and Mars. AIAA Paper 2006-6745

Prado AFBA (1997) Powered swing-by. J Guid Cont Dyn 19(5):1142-1147

Prado AFBA, Broucke RA (1995) Effects of atmospheric drag in swing-by trajectory. Acta Astron 36(6):285290

Randolph JE, McRonald AD (1992) Solar system "Fast Mission” trajectories using aerogravity assist. J Spacecr Rockets 29(2):223

Reagan F, Anandakrishnan S (1993) Dynamics of atmospheric re-entry. AIAA, Washington

Schulz W, Prado AFBA, Kuga HK, de Moraes RV (2002) Optimal space manoeuvres in a non-Keplerian force field. Adv Space Res 30(2):345-350

Silva AF, Prado AFBA, Winter OC (2013) Optimal impulsive control in a powered swing-by. In: AIAA guidance, navigation, and control (GNC) conference: Boston. AIAA guidance, navigation, and control (GNC) conference. Reston: American Institute of Aeronautics and Astronautics. vol 1, 2013

Silva AF, Prado AFBA, Winter OC (2013) Powered swing-by maneuvers around the Moon. J Phys Conf Series (Print), vol 465, p 012001, 2013

Silva AF, Prado AFBA, Winter OC (2015) A numerical study of powered swing-bys around the moon. Adv Space Res (to be published)

Sims JA, Longuski JM, Patel MR (1995) Aerogravity-assist trajectories to the outer planets. Acta Astron 35:297

Sims JA, Longuski JM, Patel MR (2000) Aerogravity-assist trajectories to the outer planets and the effect of drag. J Spacecr Rockets 37(1):49

Strange NJ, Longuski JM (2002) Graphical method for gravity-assist trajectory design. J Spacecr Rockets 39(1):9-16

Szebehely V (1973) Triple close approaches is the problem of three bodies. Celest Mech 8:163-167 
Szebehely VG (1967) Theory of orbits. Academic, New York

Tewari A (2007) Atmospheric and spaceflight dynamics. Birkhauser, Boston

Titus RR (1965) Powered flyby of Mars. Astron Acta 11(5)

Zipfel P (2007) Modeling and simulation of aerospace vehicle dynamics, 2nd edn. AIAA Education Series 2007 